\title{
USE OF TOFACITINIB IN REFRACTORY ADULT STILL DISEASE: CASE REPORT
}

Mirella Patricio Rodrigues ${ }^{1, \star}$

1.Instituto de Medicina Integral Professor Fernando Figueira, Recife (PE), Brazil.

*Corresponding author: mirella_patricio10@hotmail.com

\section{BACKGROUND}

Adult Still's disease (AOSD) is a rare, multisystem inflammatory disease. The clinical-biological picture in general includes high fever, arthritis, salmon-rash maculopapular rash, sore throat and even life-threatening complications such as macrophage activation syndrome and fulminant hepatitis. Furthermore, neutrophilic leukocytosis, hyperferritinemia and increased inflammatory evidence are salient points. Treatment is challenging with limited therapeutic options. Currently, glucocorticoids (GC), conventional synthetic DMARDs, and cytokine inhibitors targeting interleukin-1 (IL-1), interleukin 6 (IL-6), TNF are used as treatments. Anti-interleukin-1 agents are not available in Brazil. Tofacitinib, a JAK1/3 inhibitor, was shown to be effective in a patient with refractory AOSD.

\section{CASE REPORT}

Female patient, diagnosed with AOSD since 2014. At baseline, she presented fever, arthritis in hands, wrists, ankles, evanescent salmon rash, pleural effusion, significant weight loss. Altered laboratory tests: 16,100 leukocytes, without deviation, erythrocyte sedimentation rate (ESR): $61 \mathrm{~mm} / 1 \mathrm{st} \mathrm{h}$, C-reactive protein (CRP): $93 \mathrm{mg} / \mathrm{L}$. Exams were performed to exclude neoplastic, infectious and other autoimmune diseases with negative results, concluding a diagnosis of AOSD. The patient was treated with prednisone and methotrexate initially with improvement of the condition, but was replaced by leflunomide due to gastrointestinal intolerance to methotrexate. The evaluation of the instituted treatments included laboratory tests, with ERS, CRP (Figure 1) leukocyte count (leukocytes and percentage of neutrophils) in addition to clinical manifestations. Patient evolving with partial response during follow-up, requiring the use of different biological agents-initially etanercept and later tocilizumab, to which she presented a severe adverse reaction. Due to this context and the limited therapeutic arsenal available, tofacitinib was chosen. At this moment, the patient had significant synovitis in the wrists and ankles, CRP: $166 \mathrm{mg} / \mathrm{dL}$ and ESR: $93 \mathrm{~mm} / 1 \mathrm{st}$ h. After applying tofacitinib for one month, she showed partial improvement of polyarthritis. Clinical and laboratory remission was more important from month six (Figure 1), starting to present sustained remission and the need for lower doses of glucocorticoids.

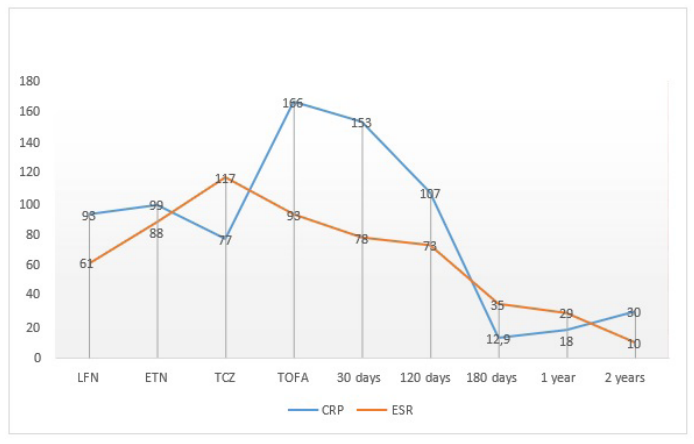

Figure 1. Erythrocyte sedimentation rate (ERS) and C-reactive protein (CRP) using tofacitinib compared to standard therapy.

\section{CONCLUSION}

The clinical case demonstrates the difficulty in treating AOSD that are refractory or intolerant to treatment with standard biological products. Anti-interleukin 1 (IL-1) agents are not available in Brazil. Tofacitinib, a JAK1/3 inhibitor, has been shown to be effective in several inflammatory diseases such as rheumatoid arthritis, systemic lupus erythematosus, and psoriasis arthritis. By blocking the pro-inflammatory effect of a wide range of cytokines, this activity may be beneficial in patients with AOSD who are refractory. Therefore, JAK inhibitors may be a new therapeutic approach for refractory AOSD.

\section{KEYWORDS}

Adult Still's disease, Tofacitinib, Multisystem inflammatory disease. 